\title{
On the Finite-Time Behaviour for Nonlinear Schrödinger Equations
}

\author{
Zhang Jian ${ }^{\star}$ \\ Department of Mathematics, Sichuan Normal University, Chengdu, 610068, P. R. China
}

Received: 23 February 1993

\begin{abstract}
Consider the nonlinear Schrödinger equation $u_{t}-i \Delta u=f(u)$. For $f(u)=$ $\pm|u|^{1+p}, \pm i|u|^{1+p}, \pm u|u|^{p}(p>0)$, and the Dirichlet boundary or nonlinear boundary (including the Neumann boundary and the Robin boundary) conditions, we establish the local estimates for the time $t$ to the solutions of the initial-boundary value problems. Being based up on these estimates, we investigate the blowing-up properties of the solutions.
\end{abstract}

\section{Introduction}

In this paper, we deal with nonlinear Schrödinger equations

$$
u_{t}-i \Delta u=f(u) \text {. }
$$

Equations of the form (1.1) arise in several areas of applied physics. In particular, they appear in nonrelativistic quantum mechanics, in the theory of superconductivity, and in the theory of laser beam propagation.

The initial value problems of (1.1) have been extensively studied. In particular, the blowing-up properties in finite time of the initial value problems and the behaviour near the blowing-up time of the solutions are given sufficient attention (see $[1-4,6])$. This is because blowing-up corresponds to the self-trapping and intense focusing of beams and mass concentration in laser beam propagation (see $[5,7])$. In the theory of superconductivity and nonrelativistic quantum mechanics, blowing-up also corresponds to the relevant physics properties.

For the initial-boundary value problems of (1.1), however, much is unknown. The purpose of this paper is to investigate the initial-boundary value problems of (1.1). Corresponding to the investigation of finite-time blowing-up properties and behaviour of the solutions near the blowing-up time to the initial value problems

\footnotetext{
$\star$ Research supported in part by the Youth Foundation of Sichuan Education Committee and the Natural Science Foudation of China
} 
of (1.1), for the initial-boundary value problems of (1.1), the questions which we consider are under which conditions, solutions of the problems blow-up in finite time, and give a description of the behaviour of the solutions in blowing-up time.

For the most elementary and important six situations of the nonlinear term $f(u)$ which are $f(u)= \pm|u|^{1+p}, f(u)= \pm i|u|^{1+p}, f(u)= \pm u|u|^{p}(p>0)$, we respectively establish the local estimates of $L^{2}$ norms of the solutions of the initial-boundary value problems of (1.1). By these local estimates, we can easily obtain the blowingup properties of the solutions. At the same time, these local estimates also describe the behaviour of the solutions near blowing-up time. It may be shown that these results are natural, simple and complete. In addition, these results can be easily generalized.

In what follows, we will first give an illustration of signs in this paper and some preparatory work. We next establish the local estimates of $L^{2}$ norms of the Dirichlet boundary value problems. Based upon these estimates, the blowing-up properties of the solutions are obtained. Finally we give remarks on the nonlinear boundary value problems (including the Neumann and Robin boundary value problems). The results for the nonlinear boundary value cases are similar to and correspond to the results for the Dirichlet boundary value cases.

In addition, for the situations of the nonlinear term $f(u)= \pm i u|u|^{p}(p>0)$, we can refer to the discussion of Kavian [9].

\section{Notation and Preparatory Work}

Now we illustrate the signs in this paper as follows:

$\partial_{t}=\frac{\partial}{\partial t}, i=\sqrt{-1} . \Omega$ is a bounded domain in $R^{n}$ with smooth boundary $\partial \Omega$. $v=v(x)$ is the outer normal vector at $x \in \partial \Omega . \Delta$ is the Laplace operator on $\Omega \subset R^{n} . u=u(t, x)$ is a complex valued function defined on $[0, T) \times \Omega$ for some $T>0 . f: C \rightarrow C$ is a complex valued function. $\nabla$ is the gradient operator.

In the domain $\Omega$, we introduce the linear eigenvalue problem of the Laplace operator :

$$
\left\{\begin{array}{l}
\Delta \varphi+\lambda \varphi=0, \quad x \in \Omega, \\
\varphi(x)=0, \quad x \in \partial \Omega .
\end{array}\right.
$$

It is well known that problem (2.1) exists for the first eigenvalue $\lambda>0$ and the corresponding first eigenfunction $\varphi(x)>0(x \in \Omega)$, moreover $\int_{\Omega} \varphi(x) d x=1$. It is obvious that $\lambda$ and $\varphi$ only relate to $\Omega$.

\section{Local Estimates}

Consider the initial-boundary value problems for nonlinear Schrödinger equation with the Dirichlet boundary value.

$$
\begin{aligned}
& \partial_{t} u-i \Delta u=f(u), \quad t>0, \quad x \in \Omega, \\
& u(0, x)=u_{0}(x), \quad x \in \Omega, \\
& u(t, x)=0, \quad t>0, \quad x \in \partial \Omega .
\end{aligned}
$$

We do not study the local existence and uniqueness of problems (3.1), (3.2), (3.3). We suppose that a local solution is given. 
Let $T>0, p>0$, and consider a solution $u$ of the problems (3.1), (3.2), (3.3) such that

$$
u=u(t, x) \in C^{1}\left([0, T), L^{2}(\Omega)\right) \cap C\left([0, T), H^{2} \cap H_{0}^{1} \cap L^{p+1}(\Omega)\right) .
$$

Then we have the following results:

Theorem 3.1. Suppose that $f(u)=i|u|^{1+p}$.

1. When $u_{0}=u_{0}(x)$ satisfies

$$
J(0)=\frac{1}{2} \int_{\Omega} \varphi(x) \operatorname{Re} u_{0} d x<0
$$

for $T \leqq \frac{\pi}{\lambda}, t \in[0, T]$, we have

$$
\begin{gathered}
\|u\|_{L^{2}(\Omega)} \geqq C_{1} /\left[1-\frac{p}{2 \lambda}|J(0)|^{p}(1-\cos \lambda t)\right]^{\frac{1}{p}}, \\
\|\nabla u\|_{L^{2}(\Omega)} \geqq C_{1}^{\prime} /\left[1-\frac{p}{2 \lambda}|J(0)|^{p}(1-\cos \lambda t)\right]^{\frac{1}{p}},
\end{gathered}
$$

where $C_{1}$ and $C_{1}^{\prime}$ are some constants.

2. When $u_{0}=u_{0}(x)$ satisfies

$$
J(0)=-\frac{1}{2} \int_{\Omega} \varphi(x) \operatorname{Im} u_{0} d x<0,
$$

for $T \leqq \frac{\pi}{2 \lambda}, t \in[0, T]$, we have

$$
\begin{gathered}
\|u\|_{L^{2}(\Omega)} \geqq C_{2} /\left[1-\frac{p}{2 \lambda}|J(0)|^{p} \sin \lambda t\right]^{\frac{1}{p}}, \\
\|\nabla u\|_{L^{2}(\Omega)} \geqq C_{2}^{\prime} /\left[1-\frac{p}{2 \lambda}|J(0)|^{p} \sin \lambda t\right]^{\frac{1}{p}},
\end{gathered}
$$

where $C_{2}$ and $C_{2}^{\prime}$ are some constants.

Theorem 3.2. Suppose that $f(u)=|u|^{1+p}$.

1. When $u_{0}=u_{0}(x)$ satisfies

$$
J(0)=\frac{1}{2} \int_{\Omega} \varphi(x) \operatorname{Re} u_{0} d x>0,
$$

for $T \leqq \frac{\pi}{2 \lambda}, t \in[0, T]$, we have

$$
\begin{gathered}
\|u\|_{L^{2}(\Omega)} \geqq C_{2} /\left[1-\frac{p}{2 \lambda}(J(0))^{p} \sin \lambda t\right]^{\frac{1}{p}}, \\
\|\nabla u\|_{L^{2}(\Omega)} \geqq C_{2}^{\prime} /\left[1-\frac{p}{2 \lambda}(J(0))^{p} \sin \lambda t\right]^{\frac{1}{p}},
\end{gathered}
$$

where $C_{2}$ and $C_{2}^{\prime}$ are some constants.

2. When $u_{0}=u_{0}(x)$ satisfies

$$
J(0)=-\frac{1}{2} \int_{\Omega} \varphi(x) \operatorname{Im} u_{0} d x<0,
$$


for $T \leqq \frac{\pi}{\lambda}, t \in[0, T]$, we have

$$
\begin{gathered}
\|u\|_{L^{2}(\Omega)} \geqq C_{1} /\left[1-\frac{p}{2 \lambda}|J(0)|^{p}(1-\cos \lambda t)\right]^{\frac{1}{p}}, \\
\|\nabla u\|_{L^{2}(\Omega)} \geqq C_{1}^{\prime} /\left[1-\frac{p}{2 \lambda}|J(0)|^{p}(1-\cos \lambda t)\right]^{\frac{1}{p}},
\end{gathered}
$$

where $C_{1}$ and $C_{1}^{\prime}$ are some constants.

Theorem 3.3. Suppose that $f(u)=-i|u|^{1+p}$

1. When $u_{0}=u_{0}(x)$ satisfies

$$
J(0)=\frac{1}{2} \int_{\Omega} \varphi(x) \operatorname{Re} u_{0} d x>0,
$$

for $T \leqq \frac{\pi}{\lambda}, t \in[0, T]$, we have

$$
\begin{gathered}
\|u\|_{L^{2}(\Omega)} \geqq C_{1} /\left[1-\frac{p}{2 \lambda}(J(0))^{p}(1-\cos \lambda t)\right]^{\frac{1}{p}}, \\
\|\nabla u\|_{L^{2}(\Omega)} \geqq C_{1}^{\prime} /\left[1-\frac{p}{2 \lambda}(J(0))^{p}(1-\cos \lambda t)\right]^{\frac{1}{p}},
\end{gathered}
$$

where $C_{1}, C_{1}^{\prime}$ are some constants.

2. When $u_{0}=u_{0}(x)$ satisfies

$$
J(0)=-\frac{1}{2} \int_{\Omega} \varphi(x) \operatorname{Im} u_{0} d x>0,
$$

for $T \leqq \frac{\pi}{2 \lambda}, t \in[0, T]$, we have

$$
\begin{gathered}
\|u\|_{L^{2}(\Omega)} \geqq C_{2} /\left[1-\frac{p}{2 \lambda}(J(0))^{p} \sin \lambda t\right]^{\frac{1}{p}}, \\
\|\nabla u\|_{L^{2}(\Omega)} \geqq C_{2}^{\prime} /\left[1-\frac{p}{2 \lambda}(J(0))^{p} \sin \lambda t\right]^{\frac{1}{p}},
\end{gathered}
$$

where $C_{2}, C_{2}^{\prime}$ are some constants.

Theorem 3.4. Suppose that $f(u)=-|u|^{1+p}$.

1. When $u_{0}=u_{0}(x)$ satisfies

$$
J(0)=\frac{1}{2} \int_{\Omega} \varphi(x) \operatorname{Re} u_{0} d x<0,
$$

for $T \leqq \frac{\pi}{2 \lambda}, t \in[0, T]$, we have

$$
\begin{gathered}
\|u\|_{L^{2}(\Omega)} \geqq C_{2} /\left[1-\frac{p}{2 \lambda}|J(0)|^{p} \sin \lambda t\right]^{\frac{1}{p}}, \\
\|\nabla u\|_{L^{2}(\Omega)} \geqq C_{2}^{\prime} /\left[1-\frac{p}{2 \lambda}|J(0)|^{p} \sin \lambda t\right]^{\frac{1}{p}},
\end{gathered}
$$

where $C_{2}$ and $C_{2}^{\prime}$ are some constants. 
2. When $u_{0}=u_{0}(x)$ satisfies

$$
J(0)=-\frac{1}{2} \int_{\Omega} \varphi(x) \operatorname{Im} u_{0} d x>0,
$$

for $T \leqq \frac{\pi}{\lambda}, t \in[0, T]$, we have

$$
\begin{gathered}
\|u\|_{L^{2}(\Omega)} \geqq C_{1} /\left[1-\frac{p}{2 \lambda}(J(0))^{p}(1-\cos \lambda t)\right]^{\frac{1}{p}}, \\
\|\nabla u\|_{L^{2}(\Omega)} \geqq C_{1}^{\prime} /\left[1-\frac{p}{2 \lambda}(J(0))^{p}(1-\cos \lambda t)\right]^{\frac{1}{p}},
\end{gathered}
$$

where $C_{1}$ and $C_{1}^{\prime}$ are some constants.

Theorem 3.5. Suppose that $f(u)=u|u|^{p}$ or $f(u)=-u|u|^{p}$. When $u_{0}=u_{0}(x)$ satisfies

$$
\int_{\Omega}\left|u_{0}(x)\right|^{2} d x \neq 0
$$

for $t \in[0, T]$, we have

$$
\begin{aligned}
\|u\|_{L^{2}(\Omega)} & \geqq C_{0} /\left[T_{0}-t\right]^{\frac{1}{p}}, \\
\|\nabla u\|_{L^{2}(\Omega)} & \geqq C_{0}^{\prime} /\left[T_{0}-t\right]^{\frac{1}{p}},
\end{aligned}
$$

where $C_{0}$ and $C_{0}^{\prime}$ are some constants, $T_{0}=\left[p\left(\int_{\Omega} d x\right)^{-\frac{p}{2}}\left(\int_{\Omega}\left|u_{0}\right|^{2} d x\right)^{\frac{p}{2}}\right]^{-1}$.

Obviously, the above theorems give a description of behaviour of the solutions in finite time for problems (3.1), (3.2), (3.3). In what follows, we prove these theorems.

Proof of Theorem 3.1. We first prove 1. For the solution $u(t, x)$ of problems (3.1), (3.2), (3.3) satisfying (3.4), let

$$
J(t)=\frac{1}{4} \int_{\Omega}\left[e^{i \lambda t} u+e^{-i \lambda t} \bar{u}\right] \varphi(x) d x,
$$

where $\lambda$ and $\varphi(x)$ are the eigenvalue and the eigenfunction respectively on the domain $\Omega$ such that (2.1), and $t \in[0, T], T \leqq \frac{\pi}{\lambda}$. Thus

$$
J^{\prime}(t)=\frac{i \lambda}{4} \int_{\Omega}\left[e^{i \lambda t} u-e^{-i \lambda t} \bar{u}\right] \varphi(x) d x+\frac{1}{4} \int_{\Omega}\left[e^{i \lambda t} \partial_{t} u+e^{-i \lambda t} \partial_{t} \bar{u}\right] \varphi(x) d x .
$$

Because

$$
\begin{aligned}
\int_{\Omega} \partial_{t} u \varphi(x) d x & =\int_{\Omega}[i \Delta u+f(u)] \varphi(x) d x=i \int_{\Omega} u \Delta \varphi d x+\int_{\Omega} f(u) \varphi(x) d x \\
& =-i \lambda \int_{\Omega} u \varphi d x+i \int_{\Omega}|u|^{1+p} \varphi(x) d x,
\end{aligned}
$$

it follows that

$$
\int_{\Omega} \partial_{t} \bar{u} \varphi(x) d x=i \lambda \int_{\Omega} \bar{u} \varphi(x) d x-i \int_{\Omega}|u|^{1+p} \varphi(x) d x
$$


Therefore, inserting (3.6), (3.7) into (3.5) and computing, we have

$$
J^{\prime}(t)=-\frac{1}{2} \sin (\lambda t) \int_{\Omega}|u|^{1+p} \varphi(x) d x .
$$

In view of Hölder's inequality,

$$
\begin{aligned}
\int_{\Omega}|u|^{1+p} \varphi(x) d x & \geqq\left(\int_{\Omega} \varphi(x) d x\right)^{-p}\left(\int_{\Omega}|u \varphi(x)| d x\right)^{1+p} \\
& =\left(\int_{\Omega}|u| \varphi(x) d x\right)^{1+p} .
\end{aligned}
$$

Since

$$
\begin{aligned}
|u| & =\sqrt{(\operatorname{Re} u)^{2}+(\operatorname{Im} u)^{2}} \geqq \frac{|\operatorname{Re} u|+|\operatorname{Im} u|}{2} \\
& \geqq \frac{|\operatorname{Re} u||\cos \lambda t|+|\operatorname{Im} u||\sin \lambda t|}{2} \geqq \frac{1}{4}\left|e^{i \lambda t} u+e^{-i \lambda t} \bar{u}\right|,
\end{aligned}
$$

inserting the above inequality into (3.9), we imply that

$$
\begin{aligned}
\int_{\Omega}|u|^{1+p} \varphi(x) d x & \geqq\left[\int_{\Omega}^{1} \frac{1}{4}\left|e^{i \lambda t} u+e^{-i \lambda t} \bar{u}\right| \varphi(x) d x\right]^{1+p} \\
& \geqq\left|\frac{1}{4} \int_{\Omega}\left[e^{i \lambda t} u+e^{-i \lambda t} \bar{u}\right] \varphi(x) d x\right|^{1+p}=|J(t)|^{1+p} .
\end{aligned}
$$

For $t \in[0, T]$ and $T \leqq \frac{\pi}{\lambda}$, we have $\sin \lambda t \geqq 0$. By (3.8) and (3.11), it follows that

$$
J^{\prime}(t) \leqq-\frac{1}{2} \sin (\lambda t)|J(t)|^{1+p}, \quad t \in[0, T], \quad T \leqq \frac{\pi}{\lambda} .
$$

Since

$$
J(0)=\frac{1}{4} \int_{\Omega}\left[u_{0}+\bar{u}_{0}\right] \varphi(x) d x=\frac{1}{2} \int_{\Omega} \varphi(x) \operatorname{Re} u_{0} d x<0,
$$

it follows by (3.12) that $J(t)<0, t \in[0, T]$ and $T \leqq \frac{\pi}{\lambda}$. Furthermore, in view of (3.12), we imply

$$
-J(t) \geqq\left[1-\frac{p}{2 \lambda}(-J(0))^{p}(1-\cos \lambda t)\right]^{-\frac{1}{p}}(-J(0)),
$$

where $t \in[0, T]$ and $T \leqq \frac{\pi}{\lambda}$. By the Schwartz's inequality, we get

$$
\|u\|_{L^{2}(\Omega)}=\left(\int_{\Omega}|u|^{2} d x\right)^{\frac{1}{2}} \geqq\left(\int_{\Omega}|\varphi(x)|^{2} d x\right)^{-\frac{1}{2}} \int_{\Omega}|u \varphi(x)| d x .
$$

It follows from (3.10) that

$$
\begin{aligned}
\int_{\Omega}|u \varphi(x)| d x & =\int_{\Omega}|u| \varphi(x) d x \geqq \frac{1}{4} \int_{\Omega}\left|e^{i \lambda t} u+e^{-i \lambda t} \bar{u}\right| \varphi(x) d x \\
& \geqq \frac{1}{4}\left|\int_{\Omega}\left[e^{i \lambda t} u+e^{-i \lambda t} \bar{u}\right] \varphi(x) d x\right|=|J(t)|=-J(t) .
\end{aligned}
$$


Hence, combining with (3.14), (3.12), we obtain

$$
\|u\|_{L^{2}(\Omega)} \geqq\left(\int_{\Omega}|\varphi(x)|^{2} d x\right)^{-\frac{1}{2}}(-J(0)) /\left[1-\frac{p}{2 \lambda}(-J(0))^{p}(1-\cos \lambda t)\right]^{\frac{1}{p}},
$$

where $t \in[0, T], T \leqq \frac{\pi}{\lambda}$. Set $C_{1}=\left[\int_{\Omega}(\varphi(x))^{2} d x\right]^{-\frac{1}{2}}|J(0)|$, then

$$
\|u\|_{L^{2}(\Omega)} \geqq C_{1} /\left[1-\frac{p}{2 \lambda}|J(0)|^{p}(1-\cos \lambda t)\right]^{\frac{1}{p}}, \quad t \in[0, T], \quad T \leqq \frac{\pi}{\lambda} .
$$

Since $u(t, x)=0$ when $x \in \partial \Omega$, it follows from Poincare's inequality that

$$
\|\nabla u\|_{L^{2}(\Omega)} \geqq C_{1}^{\prime} /\left[1-\frac{p}{2 \lambda}|J(0)|^{p}(1-\cos \lambda t)\right]^{\frac{1}{p}}, \quad t \in[0, T], \quad T \leqq \frac{\pi}{\lambda},
$$

where $C_{1}^{\prime}$ is some constant.

This completes the proof of 1 .

Now we prove 2. For the solution $u(t, x)$ of the problems (3.1), (3.2), (3.3) satisfying (3.4), let

$$
J(t)=\frac{i}{4} \int_{\Omega}\left[e^{i \lambda t} u-e^{-i \lambda t} \bar{u}\right] \varphi(x) d x,
$$

where $\lambda$ and $\varphi(x)$ are the eigenvalue and the eigenfunction respectively on the domain $\Omega$ such that (2.1), and $t \in[0, T], T \leqq \frac{\pi}{2 \lambda}$. Applying a similar argument as 1 , we get

$$
J^{\prime}(t) \leqq-\frac{1}{2} \cos (\lambda t)|J(t)|^{1+p}, \quad t \in[0, T], \quad T \leqq \frac{\pi}{2 \lambda} .
$$

In view of $J(0)<0$, it yields that $J(t)<0, t \in[0, T]$ and $T \leqq \frac{\pi}{2 \lambda}$. The rest of the proof is also analogous to 1 .

Proof of Theorem 3.2. 1. For the solution $u(t, x)$ of the problems (3.1), (3.2), (3.3) satisfying (3.4), let

$$
J(t)=\frac{1}{4} \int_{\Omega}\left[e^{i \lambda t} u+e^{-i \lambda t} \bar{u}\right] \varphi(x) d x,
$$

where $t \in[0, T]$ and $T \leqq \frac{\pi}{2 \lambda}$. By a similar argument as 1 in Theorem 3.1, we imply

$$
J^{\prime}(t) \geqq \frac{1}{2} \cos (\lambda t)|J(t)|^{1+p}, \quad t \in[0, T], \quad T \leqq \frac{\pi}{2 \lambda} .
$$

As $J(0)>0$, it follows that $J(t)>0, t \in[0, T]$ and $T \leqq \frac{\pi}{2 \lambda}$. The rest of the proof is still analogous to 1 in Theorem 3.1 .

2. For the solution $u(t, x)$ of the problem (3.1), (3.2), (3.3) satisfying (3.4), let

$$
J(t)=\frac{i}{4} \int_{\Omega}\left[e^{i \lambda t} u-e^{-i \lambda t} \bar{u}\right] \varphi(x) d x,
$$

where $t \in[0, T]$ and $T \leqq \frac{\pi}{\lambda}$. Using similar argument as 1 in Theorem 3.1, we get

$$
J^{\prime}(t) \leqq-\frac{1}{2} \sin (\lambda t)|J(t)|^{1+p}, \quad t \in[0, T], \quad T \leqq \frac{\pi}{\lambda} .
$$


As $J(0)<0$, it follows that $J(t)<0, t \in[0, T], T \leqq \frac{\pi}{\lambda}$. The rest of the proof is also analogous to 1 in Theorem 3.1.

Proof of Theorem 3.3. 1. For the solution $u(t, x)$ of the problems (3.1), (3.2), (3.3) satisfying (3.4), let

$$
J(t)=\frac{1}{4} \int_{\Omega}\left[e^{i \lambda t} u+e^{-i \lambda t} \bar{u}\right] \varphi(x) d x,
$$

where $t \in[0, T]$ and $T \leqq \frac{\pi}{\lambda}$. By a similar argument as 1 in Theorem 3.1, we imply

$$
J^{\prime}(t) \geqq \frac{1}{2} \sin (\lambda t)|J(t)|^{1+p}, \quad t \in[0, T], \quad T \leqq \frac{\pi}{\lambda} .
$$

As $J(0)>0$, it follows that $J(t)>0, t \in[0, T]$ and $T \leqq \frac{\pi}{\lambda}$. The rest of the proof is still analogous to 1 in Theorem 3.1 .

2. For the solution $u(t, x)$ of the problem (3.1), (3.2), (3.3) satisfying (3.4), let

$$
J(t)=\frac{i}{4} \int_{\Omega}\left[e^{i \lambda t} u-e^{-i \lambda t} \bar{u}\right] \varphi(x) d x,
$$

where $t \in[0, T]$ and $T \leqq \frac{\pi}{2 \lambda}$. Applying the similar argument as 1 in Theorem 3.1, we get

$$
J^{\prime}(t) \geqq \frac{1}{2} \cos (\lambda t)|J(t)|^{1+p}, \quad t \in[0, T], \quad T \leqq \frac{\pi}{2 \lambda} .
$$

As $J(0)>0$, it follows that $J(t)>0, t \in[0, T], T \leqq \frac{\pi}{2 \lambda}$. The rest of the proof is also analogous to 1 in Theorem 3.1.

Proof of Theorem 3.4. 1. For the solution $u(t, x)$ of the problems (3.1), (3.2), (3.3) satisfying (3.4), let

$$
J(t)=\frac{1}{4} \int_{\Omega}\left[e^{i \lambda t} u+e^{-i \lambda t} \bar{u}\right] \varphi(x) d x,
$$

where $t \in[0, T]$ and $T \leqq \frac{\pi}{2 \lambda}$. By a similar argument as 1 in Theorem 3.1, we get

$$
J^{\prime}(t) \leqq-\frac{1}{2} \cos (\lambda t)|J(t)|^{1+p}, \quad t \in[0, t], \quad T \leqq \frac{\pi}{2 \lambda} .
$$

In view of $J(0)<0$, it yields that $J(t)<0, t \in[0, T]$ and $T \leqq \frac{\pi}{2 \lambda}$. The rest of the proof is still analogous to 1 in Theorem 3.1.

2. For the solution $u(t, x)$ of the problem (3.1), (3.2), (3.3) satisfying (3.4), let

$$
J(t)=\frac{i}{4} \int_{\Omega}\left[e^{i \lambda t} u-e^{-i \lambda t} \bar{u}\right] \varphi(x) d x
$$

where $t \in[0, T]$ and $T \leqq \frac{\pi}{\lambda}$. Using the similar argument as 1 in Theorem 3.1, we get

$$
J^{\prime}(t) \geqq \frac{1}{2} \sin (\lambda t)|J(t)|^{1+p}, \quad t \in[0, T], \quad T \leqq \frac{\pi}{\lambda} .
$$

As $J(0)>0$, it follows that $J(t)>0, t \in[0, T]$ and $T \leqq \frac{\pi}{\lambda}$. The rest of the proof is also analogous to 1 in Theorem 3.1 . 
Proof of Theorem 3.5. When $f$ satisfies $f(u)=u|u|^{p}(p>0)$, for the solution $u(t, x)$ of the problem (3.1), (3.2), (3.3) satisfying (3.4), we put

$$
J(t)=\int_{\Omega}|u|^{2} d x=\int_{\Omega} u \bar{u} d x
$$

Thus

$$
J^{\prime}(t)=\int_{\Omega} u \partial_{t} \bar{u} d x+\int_{\Omega} \bar{u} \partial_{t} u d x
$$

As

$$
\int_{\Omega} u \partial_{t} \bar{u} d x=\int_{\Omega} u\left(-i \Delta \bar{u}+\bar{u}|u|^{p}\right) d x=i \int_{\Omega} \sum_{k=1}^{n}\left|\frac{\partial u}{\partial x_{k}}\right|^{2} d x+\int_{\Omega}|u|^{p+2} d x .
$$

Inserting the above into (3.15), we imply that

$$
J^{\prime}(t)=2 \int_{\Omega}|u|^{p+2} d x
$$

In view of Hölder's inequality, it follows that

$$
J^{\prime}(t) \geqq 2\left(\int_{\Omega} d x\right)^{-\frac{p}{2}}[J(t)]^{1+\frac{p}{2}}
$$

Therefore

$$
J(t) \geqq J(0) T_{0}^{\frac{2}{p}} /\left(T_{0}-t\right)^{\frac{2}{p}},
$$

where $T_{0}=\left[p\left(\int_{\Omega} d x\right)^{-\frac{p}{2}}\left(\int_{\Omega}\left|u_{0}\right|^{2} d x\right)^{\frac{p}{2}}\right]^{-1}$, and $J(0)=\int_{\Omega}\left|u_{0}\right|^{2} d x \neq 0$. Hence

$$
\|u\|_{L^{2}(\Omega)}=|J(t)|^{\frac{1}{2}} \geqq C_{0} /\left[T_{0}-t\right]^{\frac{1}{p}} \text {. }
$$

Moreover we imply

$$
\|\nabla u\|_{L^{2}(\Omega)} \geqq C_{0}^{\prime} /\left[T_{0}-t\right]^{\frac{1}{p}},
$$

where $C_{0}$ and $C_{0}^{\prime}$ are some constants.

When $f$ satisfies $f(u)=-u|u|^{p}(p>0)$, for the solution $u(t, x)$ of the problem (3.1), (3.2), (3.3) satisfying (3.4), we put

$$
J(t)=\int_{\Omega}-|u|^{2} d x=-\int_{\Omega} u \bar{u} d x
$$

The rest of the proof is analogous to the above.

\section{Blowing-up Properties}

Based upon the local estimates in the former part, the blowing-up properties of the solutions of problems (3.1), (3.2), (3.3) may be obtained as follows.

Theorem 4.1. Suppose that $f(u)=i|u|^{1+p}, p>0$.

1. When $u_{0}$ satisfies

$$
J(0)=\frac{1}{2} \int_{\Omega} \varphi(x) \operatorname{Re} u_{0} d x \leqq-\left(\frac{\lambda}{p}\right)^{\frac{1}{p}},
$$


the solutions of problems (3.1), (3.2), (3.3) blow up in some finite time $T^{*} \leqq T_{0}=$ $\frac{1}{\lambda} \arccos \left(1-\frac{2 \lambda}{p|J(0)| p}\right)$. And the solution on $\left[0, T^{*}\right)$ satisfies the estimates of 1 in Theorem 3.1.

2. When $u_{0}$ satisfies

$$
J(0)=-\frac{1}{2} \int_{\Omega} \varphi(x) \operatorname{Im} u_{0} d x \leqq-\left(\frac{2 \lambda}{p}\right)^{\frac{1}{p}},
$$

the solutions of problems (3.1), (3.2), (3.3) blow up in some finite time $T^{*} \leqq T_{0}=$ $\frac{1}{\lambda} \arcsin \frac{2 \lambda}{p|J(0)|^{p}}$. And the solution on $\left[0, T^{*}\right)$ satisfies the estimates of 2 in Theorem 3.1.

Theorem 4.2. Suppose that $f(u)=|u|^{1+p}, p>0$.

1. When $u_{0}$ satisfies

$$
J(0)=\frac{1}{2} \int_{\Omega} \varphi(x) \operatorname{Re} u_{0} d x \geqq\left(\frac{2 \lambda}{p}\right)^{\frac{1}{p}},
$$

the solutions of problems (3.1), (3.2), (3.3) blow up in some finite time $T^{*} \leqq T_{0}=$ $\frac{1}{\lambda} \arcsin \frac{2 \lambda}{p|J(0)|^{p}}$. And the solution on $\left[0, T^{*}\right)$ satisfies the estimates of 1 in Theorem 3.2.

2. When $u_{0}$ satisfies

$$
J(0)=-\frac{1}{2} \int_{\Omega} \varphi(x) \operatorname{Im} u_{0} d x \leqq-\left(\frac{\lambda}{p}\right)^{\frac{1}{p}},
$$

the solutions of problems (3.1), (3.2), (3.3) blow up in some finite time $T^{*} \leqq T_{0}=$ $\frac{1}{\lambda} \arccos \left(1-\frac{2 \lambda}{p|J(0)| p}\right)$. And the solution on $\left[0, T^{*}\right)$ satisfies the estimates of 2 in Theorem 3.2.

Theorem 4.3. Suppose that $f(u)=-i|u|^{1+p}, p>0$.

1. When $u_{0}$ satisfies

$$
J(0)=\frac{1}{2} \int_{\Omega} \varphi(x) \operatorname{Re} u_{0} d x \geqq\left(\frac{\lambda}{p}\right)^{\frac{1}{p}},
$$

the solutions of problems (3.1), (3.2), (3.3) blow up in some finite time $T^{*} \leqq T_{0}=$ $\frac{1}{\lambda} \arccos \left(1-\frac{2 \lambda}{p(J(0))^{p}}\right)$. And the solution on $\left[0, T^{*}\right)$ satisfies the estimates of 1 in Theorem 3.3.

2. When $u_{0}$ satisfies

$$
J(0)=-\frac{1}{2} \int_{\Omega} \varphi(x) \operatorname{Im} u_{0} d x \geqq\left(\frac{2 \lambda}{p}\right)^{\frac{1}{p}},
$$

the solutions of problems (3.1), (3.2), (3.3) blow up in some finite time $T^{*} \leqq T_{0}=$ $\frac{1}{\lambda} \arcsin \frac{2 \lambda}{p|J(0)|^{p}}$. And the solution on $\left[0, T^{*}\right)$ satisfies the estimates of 2 in Theorem 3.3. 
Theorem 4.4. Suppose that $f(u)=-|u|^{1+p}$.

1. When $u_{0}$ satisfies

$$
J(0)=\frac{1}{2} \int_{\Omega} \varphi(x) \operatorname{Re} u_{0} d x \leqq-\left(\frac{2 \lambda}{p}\right)^{\frac{1}{p}},
$$

the solutions of problems (3.1), (3.2), (3.3) blow up in some finite time $T^{*} \leqq T_{0}=$ $\frac{1}{\lambda} \arcsin \frac{2 \lambda}{p|J(0)|^{p}}$. And the solution on $\left[0, T^{*}\right)$ satisfies the estimates of 1 in Theorem 3.4 .

2. When $u_{0}$ satisfies

$$
J(0)=-\frac{1}{2} \int_{\Omega} \varphi(x) \operatorname{Im} u_{0} d x \geqq\left(\frac{\lambda}{p}\right)^{\frac{1}{p}},
$$

the solutions of problems (3.1), (3.2), (3.3) blow up in some finite time $T^{*} \leqq T_{0}=$ $\frac{1}{\lambda} \arccos \left(1-\frac{2 \lambda}{p|J(0)|^{p}}\right)$. And the solution on $\left[0, T^{*}\right)$ satisfies the estimates of 2 in Theorem 3.4.

Theorem 4.5. Suppose that $f(u)=u||^{p}$ or $f(u)=-u|u|^{p}, p>0$. When $u_{0}$ satisfies $\int_{\Omega}\left|u_{0}\right|^{2} d x \neq 0$, the solutions of problems (3.1), (3.2), (3.3) blow up in some finite time $T^{*} \leqq T_{0}=\left[p\left(\int_{\Omega} 1 d x\right)^{-\frac{p}{2}}\left(\int_{\Omega}\left|u_{0}\right|^{2} d x\right)^{\frac{p}{2}}\right]^{-1}$. And the solution on $\left[0, T^{*}\right)$ satisfies the estimates in Theorem 3.5 .

Now we prove the above theorems.

Proof of Theorem 4.1. 1. In view of the condition which $u_{0}$ satisfies, for $T_{0}=$ $\frac{1}{\lambda} \arccos \left(1-\frac{2 \lambda}{p|J(0)|^{p}}\right)$, it follows that $T_{0} \leqq \frac{\pi}{\lambda}$. Therefore, if $T_{0} \leqq T \leqq \frac{\pi}{\lambda}$, by 1 in Theorem 3.1 , it follows that

$$
\begin{aligned}
\lim _{t \rightarrow \tau_{0}^{-}}\|u\|_{L^{2}(\Omega)} & =\infty, \\
\lim _{t \rightarrow \tau_{0}^{-}}\|\nabla u\|_{L^{2}(\Omega)} & =\infty .
\end{aligned}
$$

Hence we see that $T<T_{0}$. That is, the solutions of problems (3.1), (3.2), (3.3) blow up in some finite time $T^{*} \leqq T_{0}$. And the solution on $\left[0, T^{*}\right)$ satisfies the estimates of 1 in Theorem 3.1 .

The proof of 2 is similar to 1 .

Proofs of Theorems 4.2, 4.3, 4.4 and 4.5 are analogous to the proof of Theorem 4.1.

\section{Remarks on the Nonlinear Boundary Value Problems}

Consider the initial boundary value problems for nonlinear Schrödinger equations with the nonlinear boundary:

$$
\begin{aligned}
\partial_{t} u-i \Delta u & =f(u), \quad t>0, \quad x \in \Omega, \\
u(0, x) & =u_{0}(x), \quad x \in \Omega,
\end{aligned}
$$




$$
\frac{\partial u}{\partial v}=g(t, x, u), \quad t \geqq 0, \quad x \in \partial \Omega,
$$

where $g(t, x, u)$ is a complex valued function of $n+2$ variables.

When $g(t, x, u) \equiv 0$, the problems (5.1), (5.2), (5.3) are the initial boundary value problems with the Neumann boundary.

For some $\sigma(x) \geqq 0(x \in \partial \Omega)$, when $g(t, x, u)=-\sigma(x) u$, the problems (5.1), (5.2), (5.3) are the initial boundary value problem with Robin boundary.

In the domain $\Omega$, we introduce the linear eigenvalue problems of the Laplace operator:

$$
\left\{\begin{array}{l}
\Delta \psi+\mu \psi=0, \quad x \in \Omega, \\
\frac{\partial \psi(x)}{\partial v}+\sigma(x) \psi(x)=0, \quad x \in \partial \Omega,
\end{array}\right.
$$

where $\sigma(x)$ is an arbitrary nonnegative function of $x \in \partial \Omega$. It is well known that problem (5.4) exists for the first eigenvalue $\mu>0$ and the corresponding first eigenfunction $\psi(x)>0(x \in \Omega)$; moreover $\int_{\Omega} \psi(x) d x=1$. If and only if $\sigma(x) \equiv 0(x \in \partial \Omega), \mu=0$. It is obvious that $\mu$ and $\psi$ only relate to $\Omega$ and $\sigma(x)$.

Based upon the above, using a similar argument as we research the Dirichlet boundary value problems, we investigate the problems (5.1), (5.2), (5.3). Thus the results which are completely analogous to the results of the Dirichlet boundary value problems may be obtained. And the discussion on $g(t, x, u)$ includes the Neumann boundary and the Robin boundary cases.

\section{References}

1. Cazenave, T., Weissler, F.B.: The Cauchy problem for the critical nonlinear Schrödinger equation in $H^{s}$. Nonlinear Analysis (10) 14, 807-836 (1990)

2. Merle, F., Tsutsumi, Y.: $L^{2}$ concentration of blow-up solution for the nonlinear Schrödinger equation with critical power nonlinearity. J. Diff. Eqns. 84, 205-214 (1990)

3. Merle, F: Limit of the solution of a nonlinear Schrödinger equation at blow-up time. J. Funct. Anal. 84, 201-214 (1989)

4. Weinstein, M.I.: On the structure and formation of singularities in solutions to nonlinear dispersive evolution equations. Commun. Partial Diff. Eqs. 11(5), 545-565 (1986)

5. Relley, P.L.: Self-focusing of optical beams. Phys. Rev. Lett. 15, 1005-1008 (1965)

6. Glassey, R.T.: On the blowing-up of solutions to the Cauchy problem for nonlinear Schrödinger equations. J. Math. Phys. 18, 1994-1998 (1977)

7. Suydam, B.R.: Self-focusing of very powerful laser beams. J. Quantum Electron 10, 837-843 (1974)

8. Zhang, Jian: On the blowing-up properties of solution of the mixed problems for nonlinear Schrödinger equations. Math. Appl. 4, 20-26 (1989)

9. Kavian, O.: A remark on the blowingup of solutions to the Cauchy problem for nonlinear Schrödinger equations. Trans. Am. Math. Soc. 193(1), 193-203 (1987) 